\title{
Density functionals and half-metallicity in $\mathrm{La}_{2 / 3} \mathrm{Sr}_{1 / 3} \mathrm{MnO}_{3}$
}

\author{
V. Ferrari ${ }^{* 1}$, J. M. Pruneda ${ }^{2}$, and Emilio Artacho ${ }^{3,4}$ \\ ${ }^{1}$ Cavendish Laboratory, University of Cambridge, Madingley Road, Cambridge CB3 OHE, UK \\ ${ }^{2}$ Institut de Ciencia de Materials de Barcelona, CSIC Campus U.A.B., 08193 Bellaterra, Barcelona, Spain \\ ${ }^{3}$ Department of Earth Sciences, University of Cambridge, Downing Street, Cambridge CB2 3EQ, UK \\ ${ }^{4}$ Donostia International Physics Centre, Universidad del País Vasco, 20080 San Sebastian, Spain
}

PACS 71.15.Mb, 71.15.Ap,73.20.At,75.47.Lx

The electronic structure and equilibrium geometry of $\mathrm{La}_{2 / 3} \mathrm{Sr}_{1 / 3} \mathrm{MnO}_{3}$ are studied theoretically by means of density functional calculations. The doping is treated by introducing holes and a compensating jellium background. The results for the local density approximation (LDA) agree with previous LDA calculations, with an equilibrium volume $5.3 \%$ too small and with both majority and minority spin states present at the Fermi level for the relaxed system. The generalised gradient approximation (GGA) offers a qualitatively improved description of the system, with a more realistic volume, and a half-metallic behaviour for the relaxed structure, which enables studies needing theoretical relaxation. The ideal $\mathrm{MnO}_{2}$-terminated (001) surface is then described with explicit doping.

Copyright line will be provided by the publisher

1 Introduction Ferromagnetic perovskite manganites attract much attention because of interesting properties like the colossal magnetoresistance found in some phases ${ }^{1}$ and the high degree of spin polarization of the charge carriers in other phases. The optimally doped case of $\mathrm{La}_{2 / 3} \mathrm{Sr}_{1 / 3} \mathrm{MnO}_{3}$ (LSMO) reaches complete polarisation at the Fermi level, the so-called half-metallic state. ${ }^{2}$ These features make manganites very appealing for spin-polarized current injection in new devices for spintronics, such as tunnel junctions. First-principles calculations could be very helpful in the understanding and characterisation of such devices.

Descriptions of the electronic structure of bulk $\mathrm{LaMnO}_{3}$ based compounds within density-functional theory have already been documented, ${ }^{3}$ mainly based on LDA. Electrons can be highly correlated in manganites, and it is known that the electronic characteristics of some of their phases would demand a much more explicit consideration of the non-local exchange (Hund's rule in the $d$-shell of the Mn ions) than what provided by present-day GGAs. LSMO, however, is metallic and thus much more amenable to a Kohn-Sham description, as already shown by Pickett. ${ }^{3}$

Two important limitations have been found in the LDA description of LSMO. (i) The theoretical volume obtained from LDA is underestimated by $5.3 \%$, the material losing its half-metallic character in its relaxed geometry. ${ }^{?}$ This limitation has been used to argue for the need of a treatment of correlation effects beyond LDA, such as the LDA+U approach, ${ }^{4: 5}$ even if the point is still debated. ${ }^{6}$ The flawed volume in itself does not prevent realistic studies of the bulk of the pure material, since the geometry can be taken directly from experiment. It becomes a very important limitation, however, for any more complex situation (surface, interface, defect), for which the first-principles relaxation of the structure is needed before any other property can be analysed. (ii) The Sr doping is hard to describe, demanding large unit cells and statistical sampling of configurations, or alternative alloy descriptions like the coherent-phase approximation, which enormously complicate any study.

In this work we explore the GGA description of LSMO, observing that a much better estimate of the geometry is obtained, which maintains a half-metallic character. It is important to stress that this is not

\footnotetext{
* Corresponding author: e-mail: vpf20@cam.ac.uk, Phone: +44 1223 337466, Fax: +41 1223337356
} 
a fundamental result, since we do not know whether this character is retained for the right reason. This result is important for practical purposes, since it allows the use of GGA DFT for structural optimisations of LSMO and its surfaces, interfaces, defects etc. We have also tested the performance of a homogeneous approximation to hole doping, replacing Sr substitutions by an homogeneous background of compensating charge, as recently tried in other perovskite oxides. ${ }^{7}$ Finally, the GGA description is tried on the ideal (001) surface of LSMO. The homogeneous background would have no sense for this calculation since the $\mathrm{Sr}$ substitution should be distributed only within the solid and not in vacuum. Hence, we choose simple realisations of explicit doping for this latter study.

2 Method We have performed density functional theory (DFT) ${ }^{8 ; 9}$ calculations using the SIESTA method ${ }^{10}$, which is based on pseudopotentials and numerical localized atomic orbitals as basis sets. The calculations were performed using two approximations to DFT, namely, the local density approximation ${ }^{11}$ (LDA) and the generalized gradient approximation ${ }^{12}$ (GGA), both in the spin-polarised version. For LDA we use the Ceperley-Alder exchange-correlation potential, ${ }^{13}$ whereas for the GGA calculations, we use the the Perdew-Burke-Ernzerhof (PBE) scheme. ${ }^{12}$

Core electrons were replaced by norm-conserving pseudopotentials using the scalar-relativistic TroullierMartins scheme ${ }^{14}$ in the Kleinman-Bylander factorised form, ${ }^{15}$ with nonlinear core corrections. ${ }^{16} \mathrm{La}$ and $\mathrm{Mn}$ are delicate species for pseudopotentials. The $5 s^{2} 5 p^{6} 5 d^{0} 4 f^{0}$ and $3 s^{2} 3 p^{6} 3 d^{5} 4 f^{0}$ configurations were used as reference for $\mathrm{La}$ and $\mathrm{Mn}$ respectively. Notice that the $5 s^{2}$ and $5 p^{6}$ semicore states of $\mathrm{La}$ and the corresponding $3 s^{2}$ and $3 p^{6}$ states of Mn are explicitly included in the calculations. The core radii for the $s, p, d$ and $f$ components of the pseudopotential are $1.85 \mathrm{a}_{\mathrm{o}}, 2.20 \mathrm{a}_{\mathrm{o}}, 3.10 \mathrm{a}_{\mathrm{o}}, 1.40 \mathrm{a}_{\mathrm{o}}$ for La, with a radius for the core correction $\left(r_{p c}\right)$ of $1.50 \mathrm{a}_{\mathrm{o}}$. The radii for $\mathrm{Mn}$ are $1.40 \mathrm{a}_{\mathrm{o}}, 1.90 \mathrm{a}_{\mathrm{o}}, 1.50 \mathrm{a}_{\mathrm{o}}$, and 1.90 $\mathrm{a}_{\mathrm{o}}$ with $r_{p c}=0.70 \mathrm{a}_{\mathrm{o}}$. For $\mathrm{O}$ and $\mathrm{Sr}$ we use the pseudopotentials from previous work. ${ }^{17}$ For the GGA O pseudopotential we use a slight partial core correction to avoid the GGA instability close to the nucleus. ${ }^{18}$

The basis set consists of strictly localised atomic orbitals at the double- $\zeta$ polarised level. ${ }^{19}$ The semicore states are treated at the single- $\zeta$ level. The radial shape of the orbitals is determined variationally ${ }^{17}$ under a confining pressure of $0.2 \mathrm{GPa},{ }^{20}$ using cubic bulk $\mathrm{LaMnO}_{3}$ as reference for $\mathrm{La}$ and $\mathrm{Mn}$, and using a $\mathrm{H}_{2} \mathrm{O}$ molecule for O. Pseudopotentials and the basis were tested by comparing our results for cubic $\mathrm{LaMnO}_{3}$ with those in the literature both for LDA and GGA $3 ; 6$.

The $\mathrm{Sr}$ doping is treated in two ways. Firstly, by adding the desired concentration of holes to the pristine system, plus a compensating homogeneous negative background. ${ }^{7}$ This is justified by the main finding that the doping produces mainly a change in band filling with little electronic or geometric distortion otherwise. Secondly, the results for that approximation are compared with calculations with explicit Sr doping.

3 Half-metallicity in bulk LSMO In this section we consider the effect of the density functional in the lattice constant and the half-metallic character of bulk LSMO. Although LDA is able to reproduce the half-metallic behaviour for the experimental pseudo-cubic lattice parameter ${ }^{21}\left(a_{\exp }=3.89 \AA\right.$ ), this is not the case when the theoretical equilibrium lattice constant is used $\left(a_{e q}^{L D A}=3.82 \AA\right)$, as can be observed in Fig.1 (a). The origin of the problem is the underestimation of the lattice parameter $(\sim 2 \%)$, for which the minority-spin band gap is smaller. Within GGA, however, the half-metallic character is maintained for both the experimental and the theoretical lattice constants $\left(a_{e q}^{G G A}=3.91 \AA\right)$, which display a much better agreement (an overestimation of $\sim 0.5 \%$ ) and thus allow the use of GGA for structure optimisation. The dependence of the half-metallicity on volume is further explored in Fig. 1 (b), where it is shown that for values of the lattice constant below $\sim 3.80 \AA$ the half-metallic character is lost. GGA is used in the calculations presented in the rest of the paper.

The GGA band structure of LSMO is shown in Fig. 2. The bands near the Fermi level are mainly due to hybridization of the oxygen $2 p$ and the manganese $3 d$ orbitals. The conduction bands at the Fermi level correspond to the occupied $e_{g}$ majority spin states. The peaks in the DOS at $\sim 1.5 \mathrm{eV}$ below $E_{F}$ and $\sim 2$ $\mathrm{eV}$ above $E_{F}$ correspond to the $t_{2 g}$ states for the majority (occupied) and minority (unoccupied) spins. 

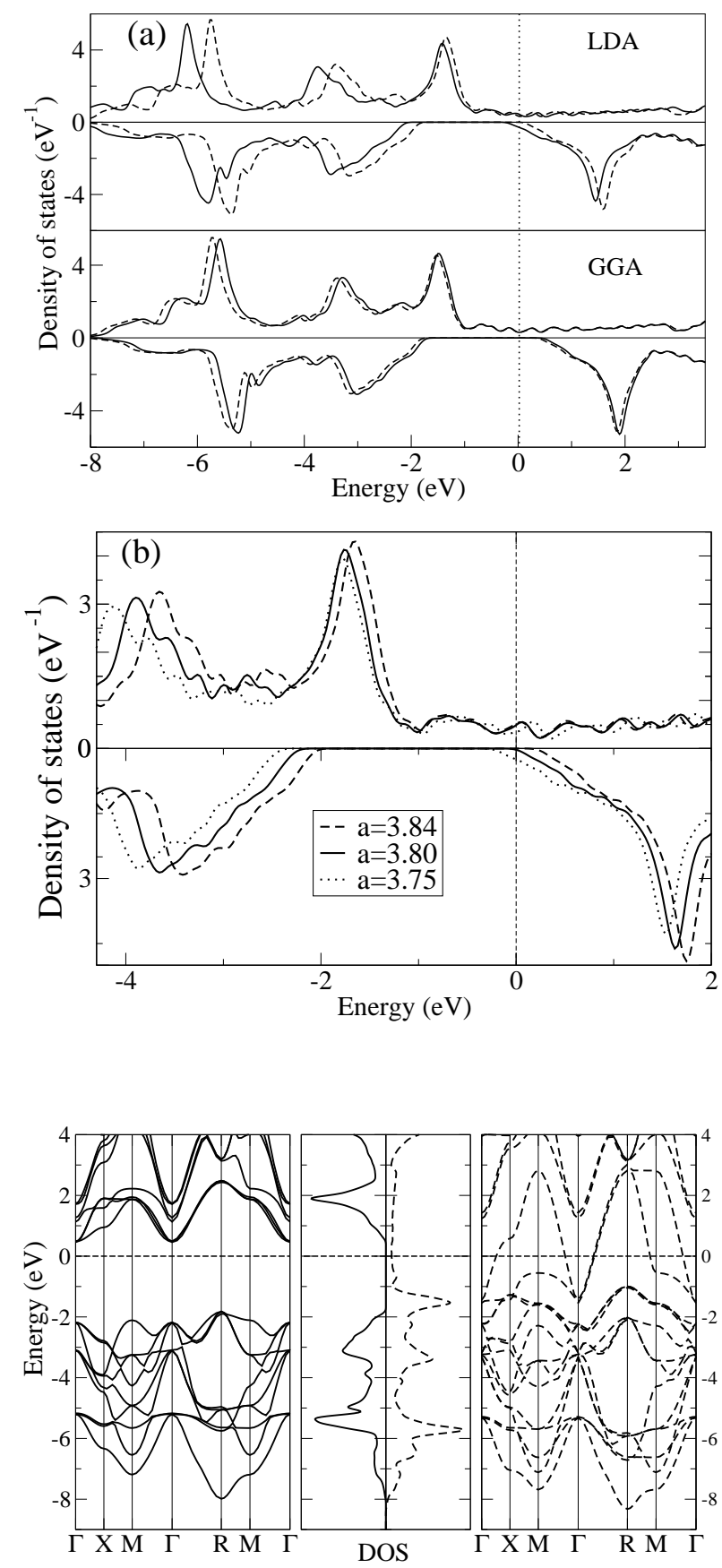

Fig. 1 (a) Comparison of the density of electronic states for the LDA and GGA approximations. Majority (minority) spin states are depicted in the upper (lower) graph of each panel. Solid lines correspond to the theoretical equilibrium lattice constant, while dashed lines are for the pseudo-cubic experimental value. (b) Density of electron states around the Fermi level for three different values of the lattice constant, within the GGA approximation.

Fig. 2 Band structure and density of states (centre panel) for hole-doped $\mathrm{LaSrMnO}_{3}$. Minority (majority) spin is shown with solid (dashed) lines.

4 Surface properties For the study of the (001) surface of LSMO we have chosen $\mathrm{MnO}_{2}$ termination. ${ }^{22}$ A slab with 6 unit cells in the perpendicular direction (12 layers of $\mathrm{MnO}_{2}-\mathrm{La}(\mathrm{Sr}) \mathrm{O}$ planes) and $4 \mathrm{x} 2$ unit cells in the parallel directions was used to model the system. The chosen thickness ensures that the bulk properties are recovered at the center of the slab, and the in-plane repetition was introduced for the description of explicit 1/3 doping, by substituting one La by Sr in every three. 
rrari: Density functionals for $\mathrm{La}_{2 / 3} \mathrm{Sr}_{1 / 3} \mathrm{MnO}_{3}$

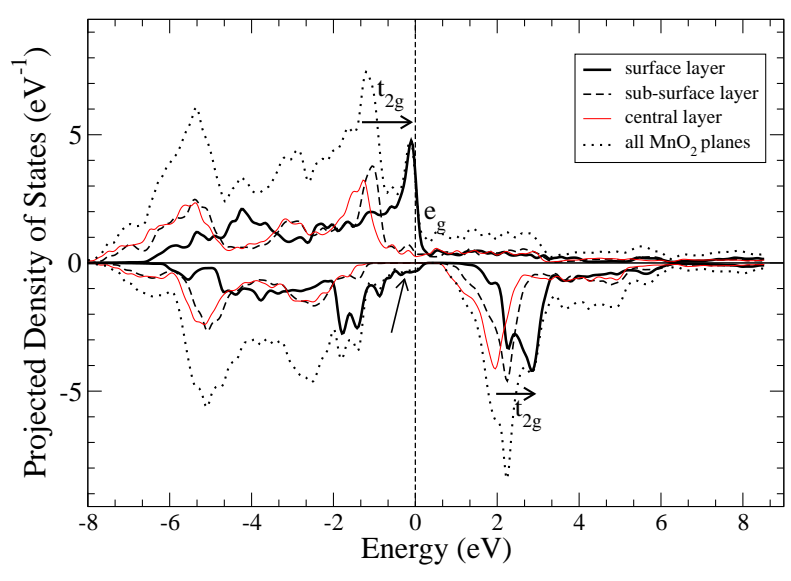

Fig. 3 Projected Density of States for different $\mathrm{MnO}_{2}$ layers in the (001) slab. Majority (minority) spin is shown in the upper (lower) panel. The shifts in the $t_{2 g}$ peaks as well as the partial occupation of the minority spin for the surface layer are highlighted by arrows.

In Fig. 3 we plot the projected density of states (PDOS) on the $\mathrm{MnO}_{2}$ layers at different depths. The PDOS on a layer is obtained by summing the PDOS for all the basis orbitals associated to that layer, and

$$
P D O S_{\mu}(E)=\sum_{n, \nu} C_{\mu n}^{*} C_{\nu n} S_{\nu \mu} \delta\left(E-E_{n}\right)
$$

where $E_{n}$ and $\psi_{n}(\vec{r})=\sum_{\mu} C_{\mu n} \phi_{\mu}(\vec{r})$ are the Kohn-Sham eigenvalues and eigenvectors, respectively, the latter expanded on the atomic basis $\left\{\phi_{\mu}(\vec{r})\right\}$, with overlap $S_{\nu \mu}$. The surface layer introduces a strong peak near the Fermi level mainly for the majority spin but with some weight on the minority spin as well that breaks the half-metallicity of the system. This deviation is very localised close to the surface. For the subsurface $\mathrm{MnO}_{2}$ layer the PDOS already resembles that of the bulk and for the central layer the halfmetallic character is fully recovered. This surface contamination of the spin polarisation was found in other calculations of doped manganites and is related to magnetic reconstructions at the surface due to changes in the electronic occupation for the $d_{z^{2}}$ and $d_{x y}$ states. ${ }^{23}$ The $t_{2 g}$ states in the surface are shifted upward by about $1 \mathrm{eV}$ and overlap with the $e_{g}$ states at the Fermi level. This changes the magnetic interaction and, although the system remains ferromagnetic, the magnetic moments near the surface are slightly reduced ( $\sim 3.4 \mu_{B}$ as compared to $\sim 3.5 \mu_{B}$ in bulk). This reduction could be related to the experimental evidence of a reduction in the magnetization at the surface of LSMO, but is in contradiction with similar theoretical calculations for doped manganites that predict an increase in the magnetic moment at the surface. ${ }^{23}$ A full characterisation of the surface state and its dependence with termination and surface relaxation will be presented elsewhere. ${ }^{24}$

5 Conclusions We have studied the bulk properties of hole-doped $\mathrm{La}_{2} / 3 \mathrm{Sr}_{1 / 3} \mathrm{MnO}_{3}$ comparing LDA and GGA (PBE). LDA gives a poor description of both the structure (lattice parameter) and the electronic properties (half-metallicity) of the system. GGA reproduces the half-metallic character and gives lattice constants in good agreement with experiment. The fact that the full (variable cell) relaxation of the bulk under GGA retains the half-metallic character allows obtaining relaxed geometries within GGA for surfaces, interfaces, or nanostructures of any sort.

Acknowledgements We thank N. Spaldin, P. Littlewood, N. Mathur, L. Hueso and L. Brey for very useful discussions. EA acknowledges the hospitality at the Donostia International Physics Centre. We acknowledge financial support from EPSRC, NERC, and BNFL.

\section{References}

[1] A. P. Ramirez, J. Phys: Condens. Matter 9, 8171 (1997). 
[2] J.-H. Park, E. Vescovo, H.-J. Kim, C. Kwon, R. Ramesh, and T. Venkatesan, Nature 392, 794 (1998).

[3] W. E. Pickett and D. J. Singh, Phys. Rev. B 53, 1146 (1996).

[4] S. Satpathy, Z. S. Popovic, and F. R. Vukajlovic, Phys. Rev. Lett. 76, 960 (1996).

[5] I. Solovyev, N. Hamada, and K. Terakura, Phys. Rev. B 53, 7158 (1996).

[6] P. Ravindran, A. Kjekshus, H. Fjellvag, A. Delin, and O. Eriksson, Phys. Rev. B 65, 064445 (2002).

[7] A. D. Walkingshaw, N. A. Spaldin, and E. Artacho, Phys. Rev. B p. 165110 (2004).

[8] P. Hohenberg and W. Kohn, Phys. Rev. pp. B864-B871 (1964).

[9] W. Kohn and L. J. Sham, Phys. Rev. pp. A1133-A1138 (1965).

[10] J. M. Soler, E. Artacho, J. D. Gale, A. García, J. Junquera, P. Ordejón, and D. Sánchez-Portal, J. Phys.: Condens. Matter 14, 2745 (2002).

[11] J. P. Perdew and A. Zunger, Phys. Rev. B 23, 5048 (1981).

[12] J. P. Perdew, K. Burke, and M. Ernzerhof, Phys. Rev. Lett. pp. 3865-3868 (1996).

[13] D. M. Ceperley and B. J. Alder, Phys. Rev. Lett. 45, 566 (1980).

[14] N. Troullier and J. L. Martins, Phys. Rev. B 43, 1993 (1991).

[15] L. Kleinman and D. M. Bylander, Phys. Rev. Lett. 48, 1425 (1982).

[16] S. G. Louie, S. Froyen, and M. L. Cohen, Phys. Rev. B 26, 1738 (1982).

[17] J. Junquera, O. Paz, D. Sánchez-Portal, and E. Artacho, Phys. Rev. B p. 235111 (2001).

[18] D. R. Hamann, Phys. Rev. Lett. pp. 660-663 (1996).

[19] E. Artacho, D. Sánchez-Portal, P. Ordejón, A. García, and J. M. Soler, Phys. Stat. Sol. (b) pp. 809-817 (1999).

[20] E. Anglada, J. M. Soler, J. Junquera, , and E. Artacho, Phys. Rev. B p. 205101 (2002).

[21] Z. El-Fadli, M. R. Metni, F. Sapina, E. Martinez, J. V. Folgado, and A. Beltran, Chemistry of Materials 14, 688 (2002).

[22] M. Yoshimoto, H. Maruta, T. Ohnishi, K. Sasaki, and H. Koinuma, Appl. Phys. Lett. 73, 187 (1998).

[23] A. Filippetti and W. E. Pickett, Phys. Rev. B 62, 11571 (2000).

[24] J. M. Pruneda, V. Ferrari, and E. Artacho. 